How language and inhibition influence analogical reasoning in children with or without developmental language disorder?

Krzemien Magali $^{a *}$, Thibaut Jean-Pierre ${ }^{\mathrm{b}}$ and Maillart Christelle ${ }^{\mathrm{a}}$

${ }^{a}$ Department of Speech and Language Therapy, Research Unit on Childhood, University of Liège, Liège, Belgium; ${ }^{b}$ LEAD CNRS UMR 5022, University of Bourgogne Franche-Comté, Dijon, France

${ }^{a}$ Department of Speech and Language Therapy, Research Unit on Childhood, University of Liège, B38, 30 rue de l'Aunaie, 4000 Liège, Belgium

${ }^{\mathrm{b}}$ LEAD CNRS UMR 5022, University of Bourgogne Franche-Comté, Pôle AAFE, 11 Esplanade Erasme, 21000 Dijon, France

*mkrzemien@uliege.be 


\section{How language and inhibition influence analogical reasoning in children with or without developmental language disorder?}

Introduction. Analogical reasoning is a human ability of crucial importance in several domains of cognition, such as numerical abilities, social cognition, and language, and which is impaired in children with developmental language disorder (DLD). This impairment might be caused by their weaknesses in inhibition or by the inefficient use of phonological recoding.

Method. We compared children with DLD and age-matched children without language disorders in an A:B::C:D analogical task. We manipulated two variables: interference (versus no interference) was used to evaluate the impact of articulatory suppression on analogical performance, and distraction (versus no distractor) was used to test whether perceptual distractors (which compete with relational responses) diminish children's performance.

Results. Contrary to expectations, articulatory suppression does not have a negative effect on analogical reasoning. In contrast, perceptual distractors have a detrimental impact on performance, and children with DLD are more impacted by perceptual distraction than their peers. Moreover, inhibition, as measured by a classical inhibition task, influences performance, but only for children with DLD.

Conclusion. The analogical reasoning impairment observed in DLD, therefore, seems to be related to perceptual distraction and inhibition rather than to phonological recoding. To conclude, this study investigates the analogical reasoning impairment observed in DLD and contributes to our understanding of the relationships between language, analogical reasoning, and executive functions.

Keywords: developmental language disorder; analogical reasoning; phonological recoding; articulatory suppression; inhibition 


\section{Introduction}

While almost all children learn language with impressive ease and speed, about $7 \%$ of children struggle with language acquisition in comprehension, expression, or both (Tomblin et al., 1997). These children are affected by developmental language disorder (DLD) [also referred to as specific language impairment (SLI)]: their nonverbal intelligence quotient does not correspond with an intellectual disability, they have no auditory deficit, no neurological disorder, and no malformation of the oral structures (Leonard, 2014). It is now well known that children with DLD also have weaknesses in domains other than language, including analogical reasoning (Leroy, Parisse, \& Maillart, 2012; Kemény \& Lukács, 2010; Vugs, Hendriks, Cuperus, \& Knoors, 2017; Yang \& Gray, 2017).

Analogical reasoning is the human ability to map two situations or domains according to their common relational structure thanks to structural alignment (Gentner \& Smith, 2012). Analogical reasoning is useful in several aspects of learning and development, such as numerical abilities (Sullivan \& Barner, 2013), social cognition (Mussweiler \& Epstude, 2009), and language (Bybee, 2010; Gentner \& Christie, 2010), or more generally in most mundane daily problem-solving situations (Hofstadter \& Sander, 2013). Analogical reasoning relies on other cognitive dimensions: working memory (i.e., the ability to temporarily store and manipulate information for complex cognitive tasks, Baddeley 1992) is used to maintain and manipulate relations (Richland, Morrison, \& Holyoak, 2006); inhibition helps to focus on relational similarities while resisting perceptual information (Richland et al., 2006; Thibaut, French, \& Vezneva, 2010).

Children with DLD may have difficulty solving not only verbal (Masterson, Evans, \& Aloia, 1993) but also nonverbal analogies (Nippold, Erskine, \& Freed, 1988), even if they have normal nonverbal intelligence (Leonard, 2014). Children with DLD have difficulty in solving analogies, especially when they have to reason about relations without the support of 
perceptual features. Leroy et al. (2012) used an analogical task with three sequences of three geometric forms, which varied along two variables: between-sequence similarity (the shapes of the three sequences were visually similar or not) and within-sequence similarity (the shapes in each sequence had zero, one or two perceptual features in common). The two groups performed similarly when within-sequence similarity was high (i.e., when each sequence contained two common features). By contrast, the performance of children with DLD decreased relative to their age-matched peers when the items in a sequence comprised of zero or one common feature. In another study, Leroy, Maillart, and Parisse (2014) also found an analogical reasoning impairment in children with DLD, either in a linguistic or in a nonlinguistic modality. Moreover, children with DLD were more affected than their age-matched peers by the absence of between-sequence similarity for the linguistic modality, in which phonological processing was involved. Children with DLD are, therefore, impaired when they have to solve verbal or nonverbal analogies. To reason analogically, children with DLD rely more on perceptual similarities than their peers. Leroy et al. (2012) mentioned the influence of inhibition to explain these weaknesses, as this function is closely related to analogical reasoning and, more specifically, to the ability to focus on relational similarity while resisting perceptual information (Richland et al., 2006; Thibaut et al., 2010).

Indeed, several causes can explain the analogical reasoning impairment observed in DLD. First, children with DLD display weaknesses in executive functions. Interestingly, Yang and Gray (2017) evaluated executive functioning in preschool-aged children with DLD in linguistic and visual modalities. Their results revealed that these children were impaired in a linguistic inhibition task (which was a child version of the Flanker task) and in updating and shifting for both modalities. Im-Bolter, Johnson, and Pascual-Leone (2006) evaluated the executive functions of school-aged children with DLD and found similar results: children 
with DLD performed worse than their age-matched peers in verbal and visual mental attention, inhibition of prepotent responses, and updating, but they had a similar performance in shifting. These deficits are also encountered in studies using questionnaires about executive functioning in daily life (Cuperus, Vugs, Scheper, \& Hendriks, 2014; Kuusisto, Nieminen, Helminen, \& Kleemola, 2017). Several studies in DLD have, therefore, reported weaknesses in executive functions, notably in inhibition, which is closely related to analogical reasoning. Some authors have considered these deficits to explain the analogical reasoning impairment observed in DLD (Leroy et al., 2012; Leroy, Maillart, \& Parisse, 2014).

However, to the best of our knowledge, other explanations of the analogical reasoning impairment in DLD have not been considered. The analogical reasoning impairment observed in DLD might result from the influence language has on analogical reasoning (Christie \& Gentner, 2014; Gentner, Simms, \& Flusberg, 2009). To further investigate this hypothesis, we manipulated a dimension of language that has not been associated with analogical reasoning in children (i.e., the use of phonological recoding). Phonological recoding is associated with the articulatory or sub-vocal rehearsal process in Baddeley's model of working memory and refers to the process by which written and visual material can be recoded into a phonological form that can be registered and maintained in the phonological store (Baddeley, 1992; Emerson \& Miyake, 2003; Norris, Butterfield, Hall, \& Page, 2018; Poloczek, Henry, Messer, \& Büttner, 2019). This relies on covert articulation and on phonological representations (Baddeley, Thomson, \& Buchanan, 1975; Balthazar, 2003; Norris et al., 2018; Poloczek et al., 2019).

Phonological recoding has been studied using the articulatory suppression paradigm. Indeed, articulatory suppression can disrupt the use of articulatory rehearsal, thus preventing participants from registering and maintaining (in the phonological store) material presented 
visually (Baddeley, 1992). Studies have shown that articulatory suppression reduces children's performance in tasks involving planning (Lidstone, Meins, \& Fernyhough, 2010), working memory, and executive functions, such as flexibility (Fatzer \& Roebers, 2012). One study has also investigated the impact of articulatory suppression on analogical reasoning: Waltz, Lau, Grewal, and Holyoak (2000) found that continuously repeating the word the led to a decrease of relational mappings in a visual scene analogy task in adult participants. Adults use the articulatory rehearsal process (and, therefore, phonological recoding) to perform analogical reasoning tasks. Waltz et al. (2000) suggested that verbal recoding is essential in analogical reasoning, even when the stimuli are pictures.

Thus, children might use phonological recoding to solve nonverbal analogies, as they do in other cognitive tasks, and as adults do for analogical reasoning. It is possible that children rely on covert articulation and on phonological representations when performing analogies based on visual material. If this is the case, the language disorders of children with DLD might impede them from using phonological recoding efficiently, which, in turn, will affect analogical reasoning. Children with DLD have been reported to have poorer phonological representations than their typically developing peers (e.g., Ramus, Marshall, Rosen, \& van der Lely, 2013). Moreover, it has been shown that covert articulation is related to articulation rate (Stigler, Lee, \& Stevenson, 1986) and that children with DLD have an impaired articulation rate (Coady \& Evans, 2008). Thus, these children could have difficulty using phonological recoding to perform cognitive tasks (Balthazar, 2003; Lidstone, Meins, \& Fernyhough, 2012), including analogical reasoning tasks.

However, it is also possible that the influence of language on analogical reasoning is situated at a more conceptual level. Some authors have shown that associating analogical items with a word referring to the relation presented allows children to solve analogies: they can focus on relational similarities, even when the task involves conflictual information, such 
as cross-mapping (Kotovsky \& Gentner, 1996). Christie and Gentner (2014) found that 3year-old children improve their performance in an analogical task when the experimenter labels the items with the words "same" or "different", according to the targeted relation. Gentner, Simms, and Flusberg (2009) also found that children improved their performance and were not influenced by perceptual matches when the relation presented was labeled. Labels (and more precisely relational labels) seem to improve children's analogical reasoning abilities. It is, therefore, likely that the relationship between analogical reasoning and language is observed through conceptual variables in addition to phonological recoding.

We have seen that DLD is associated with an analogical reasoning impairment, for verbal and nonverbal tasks (Leroy et al., 2012; Leroy, Maillart, \& Parisse, 2014; Masterson et al., 1993). Some authors (Leroy et al., 2012; Leroy, Maillart, \& Parisse, 2014) considered the deficits of inhibition as a possible explanation for the analogical reasoning impairment of children with DLD, as these functions are tightly related (Richland et al., 2006; Thibaut et al., 2010). Children with DLD have difficulty in analogical reasoning and, more specifically, in processing relational similarity without perceptual support (Leroy et al., 2012; Leroy, Maillart, \& Parisse, 2014), which could be due to their inhibition weaknesses. However, language has also been found to influence analogical reasoning (Christie \& Gentner, 2014; Gentner et al., 2009). Moreover, articulatory suppression, which impedes the use of articulatory rehearsal, and thus of phonological recoding, decreases adults' performance in a visual scene analogy task (Waltz et al., 2000). We, therefore, wanted to examine the use of phonological recoding in children with and without language disorders in an analogical reasoning task. The language deficits of children with DLD might prevent them from using phonological recoding efficiently to solve analogies. However, the relationship between language and analogical reasoning might also be situated at a more conceptual level (Christie 
\& Gentner, 2014; Gentner et al., 2009). We addressed this issue with our analogical reasoning task.

Given those findings, the objectives of this study are the following:

- To evaluate the performance of children with DLD in an analogical reasoning task compared to age-matched peers without language disorder. By comparison with previous studies, we use a nonverbal task that involves geometric shapes transformed by shape or by color and presented simultaneously.

- To examine the impact of perceptual distractors, as a deficit of inhibition could explain the analogical reasoning impairment of children with DLD. Indeed, it has been shown that performance decreased when the number of distractors increased in typically developing children (Richland et al., 2006; Thibaut et al., 2010). Given that DLD is associated with impairments in executive functions (Im-Bolter et al., 2006; Yang \& Gray, 2017), we predict an interaction between the group and the number of distractors, with a larger difference between no-distractor and distractor conditions in DLD.

- To analyze the influence of phonological recoding, thereby testing if an inefficient use of phonological recoding might explain the analogical reasoning impairment of children with DLD. We compared an articulatory suppression condition with a no suppression condition. Following Lidstone et al. (2012), we also added a motor interference condition (tapping) to control for the double task effect: we expect that articulatory suppression will have a detrimental effect on typically developing children's performance, but that this effect will be lower in children with DLD, as they may spontaneously use phonological recoding less efficiently than their peers. 
- To examine the relationship between analogical reasoning and language through conceptual variables. Given the influence of relational labels on analogical reasoning (Christie \& Gentner, 2014; Gentner et al., 2009), we expect analogical reasoning performance to be related to morphosyntax and vocabulary in children with or without DLD.

\section{Method}

\section{Participants}

Thirty-eight children from $6 ; 10$ to $13 ; 5$ years old $(\mathrm{M}=10 ; 7, \mathrm{SD}=1 ; 11)$ were recruited for the study: 19 with a DLD, and 19 without any language disorder. Children of both groups were matched by age ( \pm 6 months) and by nonverbal intellectual quotient (IQ, \pm 8 points). The groups' descriptions are given in Table 1. The groups were equal for chronological age, nonverbal IQ, and gender, but differed on socio-economic status [evaluated by the level of maternal education, from 1 (primary education) to 5 (higher education of at least 5 years)] and on all of the language measures of our language assessment.

We tested receptive vocabulary with a picture-pointing task based on words (Evaluation du Vocabulaire en Images Peabody, EVIP, Dunn, Thériault-Whalen, \& Dunn, 1993) and receptive morphosyntax with a picture-pointing task based on sentences, which is a French adaptation of the TROG (Epreuve de COmpréhension Syntaxico-SEmantique, ECOSSE, Lecocq, 1996). Regarding expression, we evaluated phonological competences with a word repetition task (or a non-word repetition task for children above 11 years old), the vocabulary with a picture-naming task, and the morphosyntactic competence with a sentence repetition task (or a sentence elicitation task for children above 11 years old) (Evaluation du 
Langage Oral, ELO, Khomsi, 2001 or Batterie langage oral, langage écrit, mémoire, attention$2^{\text {nd }}$ edition, L2MA2, Chevrie-Muller, Maillart, Simon, \& Fournier, 2010, depending on the participant's age).

Children with DLD were recruited from schools for children with special needs in the French-speaking region of Belgium. They were all undergoing a language intervention with the speech and language pathologist in their school. All children with DLD that were enrolled in the study were monolingual and had neither hearing impairment (as confirmed by audiometry) nor neurological disorder. The children were diagnosed with DLD previous to the study, but this diagnosis was confirmed by our language and cognitive evaluation: the children with DLD have a nonverbal IQ equal or superior to 80 (evaluated with the "Nonverbal Wechsler", Wechsler \& Naglieri, 2009). Even if children with DLD have long been considered as having average nonverbal intelligence, it is now acknowledged that their nonverbal IQ sometimes fall below normal limits while not being equivalent to intellectual disability (Bishop et al., 2017). Children with DLD also scored below the $10^{\text {th }}$ percentile on at least two language measures within the five domains evaluated, in agreement with Leonard's criteria for SLI (Leonard, 2014). However, the impairment was often more severe than that with a mean $\mathrm{Z}$ score of $-3.76 \sigma$ for the whole DLD group. The expressive abilities were the most impacted, with all children except one being impaired in expressive morphosyntax, as well as in expressive phonology and/or vocabulary.

Typically developing children were recruited in ordinary schools in the Frenchspeaking regions of Belgium or France. Like the children with DLD, these children were monolingual and had neither an auditory deficit nor neurological disorder. They had no language impairment, and they never repeated a grade at school. The same evaluation as the one proposed for the children with DLD was used, and they performed above the $10^{\text {th }}$ percentile for all tasks. 
Parents gave written consent for their child's participation in the study, which received the approval of the local ethics committee.

Table 1

Characteristics of the Developmental Language Disorder (DLD) and age-matched groups.

\begin{tabular}{|c|c|c|c|}
\hline & DLD group & Age-matched group & Group difference \\
\hline Gender & 10 girls; 9 boys & 4 girls; 15 boys & Fisher $p=.09$ \\
\hline $\begin{array}{l}\text { SES } \\
\text { Median }\end{array}$ & 2 & 4 & $X^{2}(4)=10.50^{*}$ \\
\hline $\begin{array}{l}\text { Age } \\
\text { Mean }(S D)\end{array}$ & $10.48(1.91)$ & $10.67(1.90)$ & $t(36)=0.31$ \\
\hline $\begin{array}{l}\text { Nonverbal IQ -WNV } \\
\text { Mean }(S D)\end{array}$ & $95.37(10.55)$ & $96.89(7.08)$ & $t(36)=0.52$ \\
\hline $\begin{array}{l}\text { Picture-pointing (words) - EVIP } \\
\text { Raw score - Mean (SD) } \\
\text { Standardized score - Mean (SD) }\end{array}$ & $\begin{array}{l}93.74(22.34) \\
89.21(16.68)\end{array}$ & $\begin{array}{l}125.10(22.06) \\
116.90(13.31)\end{array}$ & $\begin{array}{l}t(36)=4.35^{* * *} \\
t(36)=5.65^{* * *}\end{array}$ \\
\hline $\begin{array}{l}\text { Picture-pointing (sentences) - } \\
\text { ECOSSE } \\
\text { Raw score - Mean }(S D) \\
Z \text { score - Mean }(S D)\end{array}$ & $\begin{array}{l}11.63(7.83) \\
-0.86(1.21)\end{array}$ & $\begin{array}{l}6.00(3.02) \\
0.20(0.54)\end{array}$ & $\begin{array}{c}W=85.00^{* *} \\
t(24.99)=3.51^{* *}\end{array}$ \\
\hline $\begin{array}{l}\text { (Non)word repetition - } \\
\text { ELO/L2MA2 } \\
\text { Z score - Mean (SD) }\end{array}$ & $-11.71(18.71)$ & $0.62(0.82)$ & $W=352.00 * * *$ \\
\hline $\begin{array}{l}\text { Picture-naming -ELO/L2MA2 } \\
Z \text { score - Mean (SD) }\end{array}$ & $-1.91(1.34)$ & $0.28(0.70)$ & $t(27.25)=6.29 * * *$ \\
\hline $\begin{array}{l}\text { Sentence elicitation/repetition - } \\
\text { ELO/L2MA2 } \\
\text { Z score - Mean (SD) }\end{array}$ & $-3.60(1.70)$ & $0.04(0.81)$ & $t(25.80)=8.43^{* * *}$ \\
\hline
\end{tabular}

Note. SES = socio-economic status measured by maternal education with 2 meaning middle/junior high school and 4 meaning bachelor's degree, IQ = intellectual quotient, $\mathrm{SD}=$ standard deviation, $\mathrm{WNV}=$ Wechsler Non-Verbale, EVIP = Evaluation du Vocabulaire en Images Peabody, ECOSSE = Epreuve de COmpréhension Syntaxico-SEmantique, ELO = Evaluation du Langage Oral, L2MA2 = Batterie langage oral, langage écrit, mémoire, attention- $2^{\text {nd }}$ edition, $* p$ $<.05, * * p<.01, * * * p<.001$. 


\section{Materials}

We used a nonverbal analogical reasoning task created by Vezneva (2011) in the A:B::C:D format ( $\mathrm{A}$ is to $\mathrm{B}$, what $\mathrm{C}$ is to $\mathrm{D}$ ). The task is composed of two pairs of geometric shapes: the two pictures in the first pair (A and B) had close values on one dimension, either shape or color. For analogies based on shape, A and B had similar shapes, except that one was squeezed (or elongated) compared to the other. For analogies based on color, A and B had a similar color, except that one was lighter (or darker) than the other. The child had to choose the fourth picture so that the third and fourth pictures (C and D) shared the same transformation, either the same shape but squeezed (or elongated) or the same color but lighter (or darker). For example, Figure 1A shows a shape, and Figure 1B the same shape but squeezed. Figure 1C shows another shape that the child had to associate with the same shape, but squeezed, shown in Figure 1D. For each trial, there were five possible solutions: the correct response and four distractors.

Figure 1 - Example of a test item (Vezneva, 2011) comprising a shape (A) associated with the same shape, but squeezed (B), and another shape (C) that the child had to associate with the same shape, but squeezed (D).

A

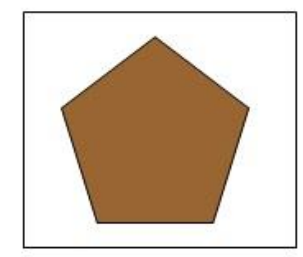

B

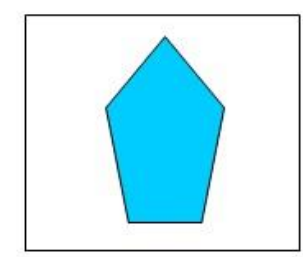

C

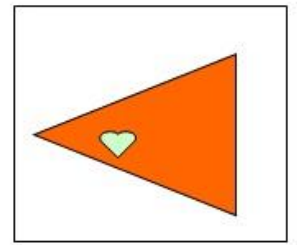

$\mathrm{D}$

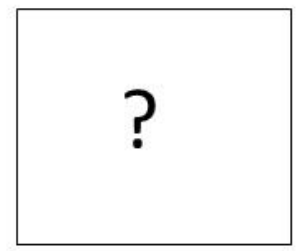

To vary the level of perceptual distraction, the items were divided into three conditions. In the no-distractor condition, none of the distractors in the array of the potential 
solutions shared a perceptual feature with the third (C) picture. In the one-distractor condition, one of the four distractors had a perceptual feature in common with the C picture (e.g., the same shape, color or small shape inside). In the three-distractor condition, three of the four distractors shared a perceptual feature with the $\mathrm{C}$ picture. An example for each condition is shown in Figure 2.

Figure 2 - Example of a test item for each condition (Vezneva, 2011): no distractor (A), one distractor (B), and three distractors (C).

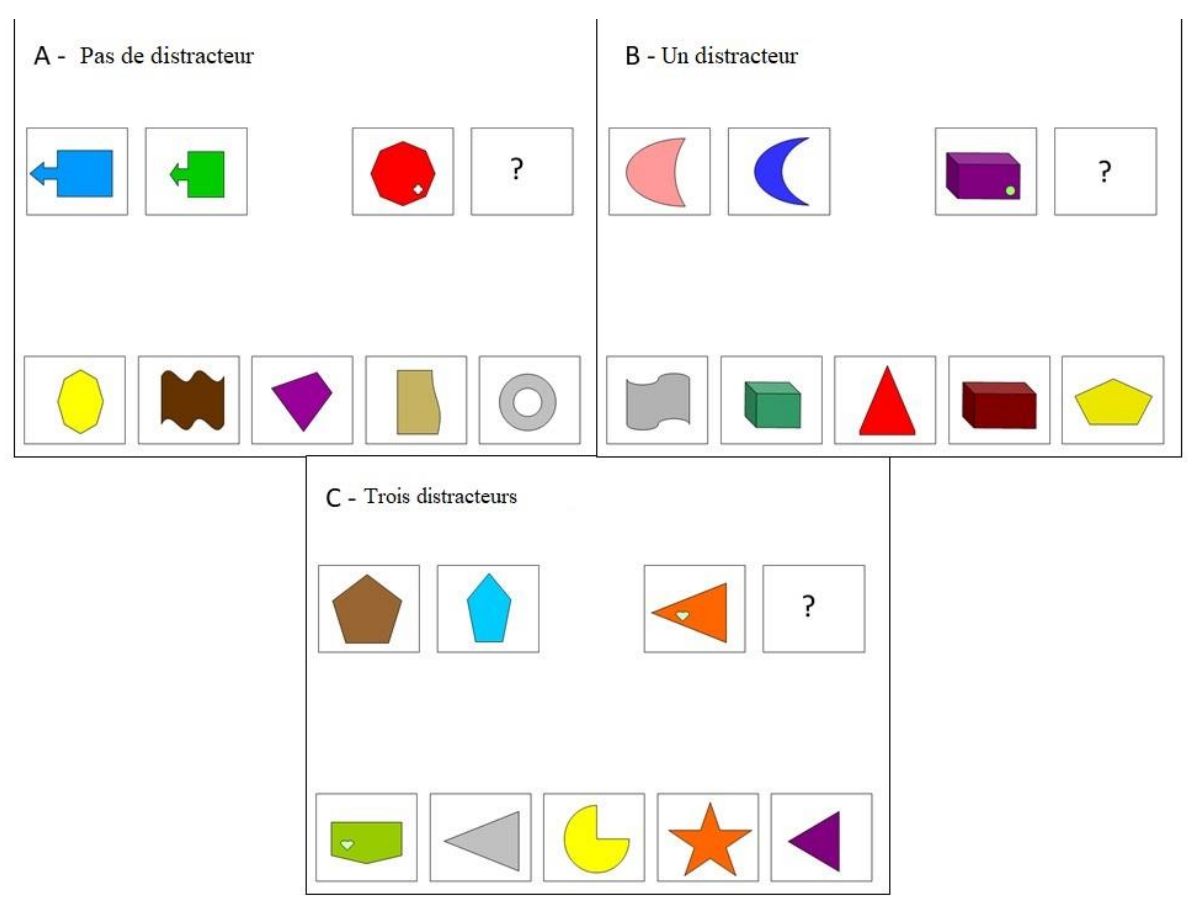

To analyze the use of language and phonological recoding to solve analogies, the trials were divided into three interference conditions: the no-interference condition, the articulatory suppression condition, and the motor interference condition. In the articulatory suppression condition, the child is asked to solve the analogy while repeating the syllable $b a$ at a pace of two syllables per second. The repetition of a Consonant-Vowel syllable at a pace of two 
syllables per second has been found to impede the use of language and phonological recoding while performing nonverbal cognitive tasks (Fatzer \& Roebers, 2012; Otsuka \& Osaka, 2015). Thus, if performance under this condition is lower than in the no-suppression condition, it means that participants usually use phonological recoding while they solve analogies. In the motor interference condition, children are asked to tap their foot against the floor at the same pace. This condition is added to control the double task effect: foot-tapping has been shown to generate the same amount of demand as the articulatory suppression task (Emerson \& Miyake, 2003). Moreover, while some authors observed a tapping deficit in children with DLD, several studies have shown that tapping is unimpaired in these children (Hill, 2001; Zelaznik \& Goffman, 2010). If the performance of children is worse under articulatory suppression than under motor interference, it cannot be imputed to the attentional cost linked to the double task situation. In the no-interference condition, participants did not perform any task other than the analogical reasoning task.

In addition to the experimental task, we also assessed participants' inhibition capacities. Indeed, this function is helpful to focus on relational similarities instead of perceptual cues (Richland et al., 2006; Thibaut et al., 2010; Thibaut, French, Vezneva, Gérard, \& Glady, 2011). Inhibition was evaluated with a 'Go-NoGo' task in which children have to push the space bar when the target stimulus appears on the screen while withholding from responding when another stimulus appears (Geurten, Catale, \& Meulemans, 2016). The inhibition measures were the percentage of correct responses and the median reaction time.

\section{Procedure}


The tasks were administered in a session of about 30 minutes, at the child's school or house. The analogical reasoning task was conducted with E-Prime 2.0 (Psychology Software Tools Inc., 2012, Sharpsburg, PA, USA) on a 15.6-inch computer monitor. The analogical reasoning task is presented within an entertaining context to encourage motivation. The instructions are the followings: "You will see a series of pictures: the first one goes with the second one, but the third one is alone, so you have to find the fourth picture to complete the series. Try with this example!". Then, the experimenter checks the comprehension of the instructions with four practice trials. If the child fails, the experimenter says: "You see, the first two pictures are slightly different, can you see this slight difference? Well, the fourth picture has to be slightly different from the third one too. So, try again!". If the child fails again, the experimenter invites him or her to focus on the shape or on the color of the pictures: "Can you see how the two first pictures are different? Yes, the second picture is bigger than the first one. So, the fourth picture has to be bigger than the third one too!".

After the practice trials, there are 18 test items, six for each of the three distraction conditions and six for the three interference conditions. Each participant must solve analogies successively without any interference, with concurrent articulatory suppression, and with concurrent foot-tapping. Before the articulatory suppression and motor interference conditions, the child is invited to repeat the syllable $b a$ or to tap his or her foot on the floor while solving the analogies. The pace is demonstrated by the experimenter. For each item, the child must press the button corresponding to the solution on the computer keyboard, as response times are measured.

The interference and distraction conditions are counterbalanced, thus leading to six versions of the task. The administration lasted about 15 minutes. 
After the analogical reasoning task, children were administered a control task to ensure that their errors were not due to an inability to perceive the transformation of the geometric shapes. This control task was also created by Vezneva (2011). Participants saw each target picture of the analogies and had to choose the picture that represents the same picture with a transformed shape/color. The mean number of correct responses in this task was $96.4 \%$, with scores ranging from $88.9 \%$ to $100 \%$, which means that a failure in the analogical task cannot be explained by difficulties in the perception of transformations. Finally, the task measuring inhibition was administered according to the standardization conditions. The administration of the control and inhibition tasks lasted about 5 to 10 minutes each.

This experiment had a $2 \times 3 \times 3$ design, with group (DLD vs. age-matched children) as a between-subject factor and interference (no interference vs. articulatory suppression vs. foot-tapping) and distraction (no distractor vs. one distractor vs. three distractors) as withinsubject factors. All of the analyses were performed in the R computing environment (2016).

\section{Results}

We first conducted an analysis of variance with the group (age-matched vs. DLD) as a between-subject factor and the type of transformation (shape vs. color) as a within-subject factor. Because we detected no difference between the two types of items, $F(1,36)=0.47, p=$ $.50, \eta^{2}{ }_{p}=.013$ for correct responses and $F(1,35)=1.95, p=.17, \eta^{2}{ }_{p}=.053$ for response times, we merged the two scores. The two groups differed on socio-economic status, so we verified if this variable had an influence on performance. We conducted an analysis of variance with the group (age-matched vs. DLD) and the socio-economic status (1 vs. 2 vs. 3 
vs. 4 vs. 5) as between-subject factors. As no effect of the socio-economic status was detected, $F(4,30)=0.35, p=.84, \eta^{2}{ }_{p}=.044$ for correct responses and $F(4,30)=0.59, p=$ $.67, \eta_{\mathrm{p}}^{2}=.073$ for response times, this variable was not included in the following analyses. We analyzed the effects of interference and distraction on correct responses and response times. Finally, we examined the correlations between our clinical, demographic, and experimental measures, and we added the inhibition measures to the models to further investigate the relationship between inhibition and analogical reasoning.

\section{The score of correct responses}

We ran a two-way mixed ANOVA with Interference (no interference vs. articulatory suppression vs. foot-tapping) and Distraction (no vs. one vs. three distractors) as withinsubject factors and Group (children with DLD vs. age-matched children) as a between-subject factor on the number of correct analogies. These data are presented in Table 2. The analysis revealed a main effect of Group, $F(1,36)=11.7, p=.002, \eta^{2} \mathrm{p}=.24$, with the children with DLD performing worse than their peers. There was no main effect of Interference, $F(2,72)=$ $0.37, p=.69, \eta_{\mathrm{p}}^{2}=.010$, and no significant interaction between Interference and Group, $F(2$, $72)=0.43, p=.65, \eta_{p}^{2}=.012$. However, there was a main effect of Distraction, $F(2,72)=$ 35.4, $p<.001, \eta^{2}{ }_{\mathrm{p}}=.50$. Post-hoc analyses using Tukey's HSD revealed that the threedistractor condition was significantly lower than the no-distractor $(p<.001)$ and onedistractor conditions $(p=.002)$. The most important result was a significant interaction between Group and Distraction, $F(2,72)=4.52, p=.014, \eta^{2} \mathrm{p}=.11$. Post-hoc analyses indicated that the effect of Distraction was mainly observed in children with DLD. As shown by Figure 3, these children performed worse in the three-distractor condition than in the nodistractor $(p<.001)$ and one-distractor conditions $(p=.031)$, while age-matched children 
seem to perform similarly in the three conditions (all $p>.13$ ). Finally, the interaction between Distraction and Interference did not reach significance, $F(4,144)=2.19, p=.073, \eta^{2} \mathrm{p}=.057$, nor did the triple interaction between Distraction, Interference, and Group, $F(4,144)=0.85, p$ $=.49, \eta^{2} \mathrm{p}=.023$.

Table 2

Proportion of correct responses (and standard deviation) as a function of Interference, Distraction and Group.

\begin{tabular}{lcccccccccc}
\hline Interference & \multicolumn{3}{c}{ No interference } & \multicolumn{3}{c}{$\begin{array}{c}\text { Articulatory } \\
\text { suppression }\end{array}$} & \multicolumn{3}{c}{ Tapping } \\
Distraction & $\mathbf{0}$ & $\mathbf{1}$ & $\mathbf{3}$ & $\mathbf{0}$ & $\mathbf{1}$ & $\mathbf{3}$ & $\mathbf{0}$ & $\mathbf{1}$ & $\mathbf{3}$ \\
\cline { 2 - 11 } Age-matched & 0.87 & 0.82 & 0.63 & 0.79 & 0.84 & 0.58 & 0.92 & 0.74 & 0.66 \\
children & $(0.28)$ & $(0.34)$ & $(0.40)$ & $(0.25)$ & $(0.24)$ & $(0.38)$ & $(0.19)$ & $(0.42)$ & $(0.41)$ \\
Children with & 0.82 & 0.37 & 0.39 & 0.74 & 0.63 & 0.32 & 0.82 & 0.53 & 0.42 \\
DLD & $(0.34)$ & $(0.37)$ & $(0.36)$ & $(0.26)$ & $(0.37)$ & $(0.30)$ & $(0.25)$ & $(0.31)$ & $(0.42)$ \\
\hline
\end{tabular}

Note. $\mathrm{DLD}=$ developmental language disorder.

Figure 3 - Proportion of correct responses as a function of Distraction and Group.

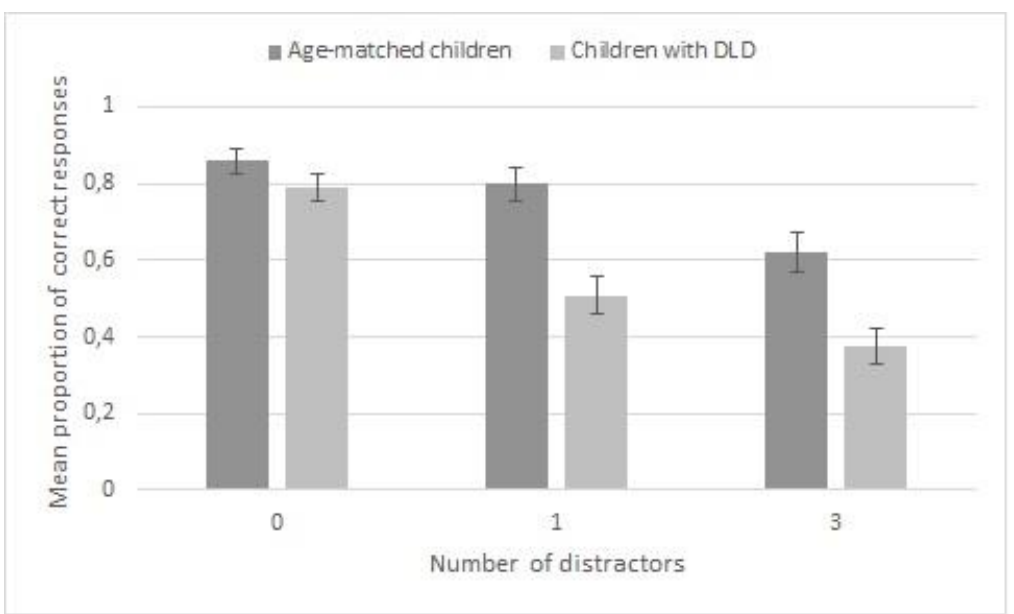

Note. $\mathrm{DLD}=$ developmental language disorders, bars represent error type. 


\section{Response times}

We ran a linear mixed-effects model on the mean response time for correct responses (we did not perform an ANOVA because we had cells with missing data due to an absence of correct responses for some participants), with Interference (no interference vs. articulatory suppression vs. foot-tapping), Distraction (no vs. one vs. three distractors), and Group (children with DLD vs. age-matched children) as fixed factors and participants as a random factor. We analyzed the effects of fixed factors with type II Wald F test using Kenward-Roger approximation. These data are presented in Table 3. The analysis revealed a significant effect of Interference, $F(2,230)=8.00, p<.001$. Post-hoc analyses (Tukey HSD) indicated that the articulatory suppression items $(\beta=-1630)$ were performed faster than the items without interference $(p=.007)$ and with tapping $(p=.002)$. No other main effect or interaction reached significance (all $p>.17, \beta$ between -2027 and 2401).

Table 3

Mean response times (and standard deviation) for correct responses in milliseconds as a function of Interference, Distraction and Group.

\begin{tabular}{|c|c|c|c|c|c|c|c|c|c|}
\hline \multirow{2}{*}{$\begin{array}{l}\text { Interference } \\
\text { Distraction }\end{array}$} & \multicolumn{3}{|c|}{ No interference } & \multicolumn{3}{|c|}{$\begin{array}{l}\text { Articulatory } \\
\text { suppression }\end{array}$} & \multicolumn{3}{|c|}{ Tapping } \\
\hline & $\mathbf{0}$ & 1 & 3 & $\mathbf{0}$ & 1 & 3 & $\mathbf{0}$ & $\mathbf{1}$ & 3 \\
\hline $\begin{array}{l}\text { Age-matched } \\
\text { children }\end{array}$ & $\begin{array}{l}7725 \\
(3357)\end{array}$ & $\begin{array}{l}7656 \\
(1915)\end{array}$ & $\begin{array}{l}6815 \\
(2661)\end{array}$ & $\begin{array}{c}6127 \\
(3432)\end{array}$ & $\begin{array}{c}6037 \\
(2308)\end{array}$ & $\begin{array}{l}7593 \\
(2382)\end{array}$ & $\begin{array}{l}7150 \\
(2741)\end{array}$ & $\begin{array}{c}7652 \\
(2848)\end{array}$ & $\begin{array}{c}7798 \\
(5875)\end{array}$ \\
\hline $\begin{array}{l}\text { Children with } \\
\text { DLD }\end{array}$ & $\begin{array}{c}8484 \\
(3841)\end{array}$ & $\begin{array}{l}10403 \\
(2863)\end{array}$ & $\begin{array}{c}7622 \\
(2828)\end{array}$ & $\begin{array}{l}6107 \\
(2570)\end{array}$ & $\begin{array}{c}6014 \\
(3148)\end{array}$ & $\begin{array}{c}6347 \\
(3328)\end{array}$ & $\begin{array}{c}8620 \\
(6048)\end{array}$ & $\begin{array}{c}8512 \\
(5061) \\
\end{array}$ & $\begin{array}{c}8677 \\
(7383)\end{array}$ \\
\hline
\end{tabular}

Note. $\mathrm{DLD}=$ developmental language disorder. 


\section{The relationship between language, inhibition, and analogical reasoning}

To investigate the relationship between our demographic, clinical, and experimental measures, we reported the correlations between socioeconomic status, language, and inhibition measures on the one hand, and response scores and response times in the analogical task on the other hand. As age has an impact on language and inhibition, we used partial correlations controlling for the effect of age. Moreover, response scores in the analogical task were positively correlated with age, $r(17)=.56, p=.013$ for children with DLD and $r(17)=$ $.50, p=.029$ for age-matched children. We used Pearson correlation and Kendall's tau coefficient when data were not normally distributed (Table 4). For children with DLD, the scores in the analogical reasoning task significantly correlated with nonverbal IQ, $r(16)=.52$, $p=.025$. For age-matched children, analogical reasoning response scores were associated with expressive vocabulary, $r(16)=.56, p=.015$, and expressive morphosyntax, $r(16)=.65$, $p=.003$. This relationship between language and analogical reasoning was not encountered in children with DLD (all $p>.082, r$ between .08 and .42 ). However, some of the correlation values corresponded to a medium effect size, even if these did not reach significance (Cohen, 1988), which is probably because of the low power. This is notably the case in children with DLD for the correlation between analogical reasoning response scores and receptive vocabulary, $r(16)=.42, p=.082$, and for the correlation between analogical reasoning response scores and expressive morphosyntax, $r(16)=.37, p=.13$. Finally, response times in the analogical task did not significantly correlate with any of the measures (all $p>.064, r$ between -.28 and .44), but here again, some values corresponded to a medium effect size and might not have reached significance due to low power. 
Table 4

Partial correlations between the demographic, clinical and experimental measures controlling for the effect of age.

\begin{tabular}{|c|c|c|c|}
\hline & & $\begin{array}{c}\text { Analogical task - } \\
\text { Response score } \\
r(16)\end{array}$ & $\begin{array}{c}\text { Analogical task - } \\
\text { Response time } \\
r(16)\end{array}$ \\
\hline \multirow{10}{*}{$\begin{array}{l}\text { Age-matched } \\
\text { children }\end{array}$} & SES & .04 & .16 \\
\hline & Nonverbal IQ & .38 & .31 \\
\hline & Phonology - Z score & .38 & .09 \\
\hline & Receptive vocabulary - Raw score & .42 & .34 \\
\hline & Expressive vocabulary $-\mathrm{Z}$ score & $.56^{*}$ & .24 \\
\hline & Receptive morphosyntax - Errors & -.42 & -.28 \\
\hline & Expressive morphosyntax $-\mathrm{Z}$ score & $.65^{* *}$ & .32 \\
\hline & Inhibition (RS) - Raw score & .18 & .14 \\
\hline & Inhibition (RT) - Raw score & .45 & .25 \\
\hline & Analogical task - Response time & .30 & / \\
\hline \multirow{10}{*}{ Children with } & SES & .23 & .01 \\
\hline & Nonverbal IQ & $.52 *$ & .32 \\
\hline & Phonology - Z score & .08 & .01 \\
\hline & Receptive vocabulary - Raw score & .42 & .44 \\
\hline & Expressive vocabulary $-\mathrm{Z}$ score & -.14 & .28 \\
\hline & Receptive morphosyntax - Errors & -.22 & -.14 \\
\hline & Expressive morphosyntax $-\mathrm{Z}$ score & .37 & .21 \\
\hline & Inhibition (RS) - Raw score & .05 & .11 \\
\hline & Inhibition (RT) - Raw score & -.19 & .07 \\
\hline & Analogical task - Response time & .32 & / \\
\hline
\end{tabular}

Note. $\mathrm{SES}=$ socio-economic status, $\mathrm{IQ}=$ intellectual quotient, $\mathrm{RS}=$ response score, $\mathrm{RT}=$ response time, $* p<.05, * * p<.01$. 
Perceptual distraction decreases response scores, especially for children with DLD. To further investigate the relationship between analogical reasoning and perceptual distraction, we set out to test if performance was predicted by inhibition, as measured by a classical GoNoGo task (Geurten et al., 2016), and if inhibition could account for the distraction effect. Inhibition has indeed been related to the ability to focus on relational similarities while resisting perceptual cues (Richland et al., 2006; Thibaut et al., 2010, 2011). As the distraction effect was mainly observed in children with DLD, we performed a separate analysis for children with DLD. Our inhibition measures were the percentage of correct responses and the median reaction time obtained in the Go-NoGo task (Geurten et al., 2016).

We conducted an analysis of covariance with Interference (no interference vs. articulatory suppression vs. foot-tapping) and Distraction (no vs. one vs. three distractors) as within-subject factors and the two inhibition measures as covariates on the mean percentage of correct responses of children with DLD. Importantly, results indicated a significant effect of inhibition reaction time, $F(1,16)=4.65, p=.046, \eta^{2} \mathrm{p}=.22$. There was no significant effect of inhibition correct responses, $F(1,16)=2.77, p=.11, \eta^{2} \mathrm{p}=.15$. Moreover, the effect of Distraction remained significant, $F(2,36)=26.8, p<.001, \eta^{2}{ }_{p}=.60$. The main effect of Interference, $F(2,36)=0.49, p=.62, \eta^{2} \mathrm{p}=.026$, and the interaction between Distraction and Interference, $F(4,72)=2.09, p=.091, \eta_{p}^{2}=.10$, did not reach significance. In summary, crucially, inhibition reaction time (as measured by a classical Go-NoGo task) explained the percentage of correct responses of children with DLD in an analogical task with different levels of perceptual distraction, which was not the case for age-matched children, $F(1,16)=$ $0.55, p=.47, \eta^{2} \mathrm{p}=.033$. By contrast, there was no such effect for the response times in the analogical task in both groups (all $p>.41, \beta$ between -17.0 and 6.40). 


\section{Discussion}

In this study, we aimed to explain the analogical impairment observed in children with DLD with an inhibition weakness or inefficient use of phonological recoding. The experiment confirms that children with DLD have worse performance than age- and nonverbal IQmatched children in a nonverbal analogical reasoning task. Our data also show that impeding phonological recoding does not have a detrimental effect on performance. In contrast, perceptual distraction decreases children's performance, especially for the DLD group. Finally, inhibition, as measured by a classical task, had a significant effect on accuracy, but only for children with DLD.

This study confirms the analogical reasoning impairment that has already been observed in DLD for verbal analogies (Masterson et al., 1993), nonverbal analogies with semantic content (Krzemien, Jemel, \& Maillart, 2017), and linguistic and non-linguistic analogies without semantic content (Leroy et al., 2012; Leroy, Maillart, \& Parisse, 2014). We replicated these results with analogies based on transformation relations and simultaneously presented geometric shapes. Leroy and colleagues (Leroy et al., 2012; Leroy, Maillart, \& Parisse, 2014) have already observed difficulty with non-linguistic analogies without semantic content in children with DLD. However, in their experiments, items were presented sequentially, which might explain their results; given the processing skills limitations of children with DLD (Im-Bolter et al., 2006), it is possible that they were more impacted by the working memory load associated with sequential presentation than by the characteristics of the analogies themselves. Our results provide new evidence for a general analogical reasoning impairment, which is independent of the modality or the presentation format of the input. Our data also provide new evidence showing that children with DLD display weaknesses in cognitive functions that are not limited to the linguistic domain. DLD had indeed been 
considered as a specific impairment of language development, while the other domains of cognition were preserved (Leonard, 2014). However, it is now acknowledged that children with DLD also have weaknesses in domains other than language: they are impaired in auditory and visuospatial working memory and in attentional and executive functions (ImBolter et al., 2006; Niemi, Gundersen, Leppäsaari, \& Hugdahl, 2003; Vugs et al., 2017; Yang \& Gray, 2017). Children with DLD also have difficulty with global and fine motricity (Hill, 2001; Zelaznik \& Goffman, 2010). Finally, these children seem to be impaired in the procedural memory system, for the learning of verbal and visuomotor sequences (Lum \& Conti-Ramsden, 2013), and for probabilistic category learning tasks (Kemény \& Lukács, 2010). Our results confirm that children with DLD have difficulty in domains other than language, as they are impaired when performing verbal and nonverbal analogies.

Regarding the effect of interference and articulatory suppression on analogical reasoning, our results seem contradictory. For response scores, there is no significant effect of the interference condition. However, the response times data indicate that the articulatory suppression condition does differ from the two other conditions: children seem to respond faster in the articulatory suppression condition when the use of phonological recoding is impeded. These findings are unexpected because articulatory suppression is beneficial for both groups, whereas we had expected that it would increase response times. This result is not consistent with other studies, which found either no effect or a detrimental effect of articulatory suppression on reasoning tasks involving analogies (Rao \& Baddeley, 2013; Waltz et al., 2000). Thus, this data seems difficult to interpret. In other studies, some authors highlighted that participants were able to abandon the strategy of phonological recoding in a serial recall task if they were under an articulatory suppression condition or if they were explicitly asked to favor a semantic recoding strategy (Campoy \& Baddeley, 2008; Norris et 
al., 2018). In our study, children might have abandoned the use of phonological recoding under articulatory suppression for a strategy based on semantics or visuospatial modality (e.g., Baddeley \& Larsen, 2007; Logie, Della Sala, Wynn, \& Baddeley, 2000). These strategies might be as efficient in terms of accuracy, but phonological recoding, which requires subvocal verbalization, might require more processing and, thus, more time than the articulatory suppression condition, especially for children with DLD (Balthazar, 2003; Lidstone et al., 2012). However, it is also possible that our articulatory condition did not succeed in impeding phonological recoding. Thus, Norris et al. (2018) suggested that articulatory suppression might not block phonological recoding completely. It seems, therefore, difficult to conclude about the impact of phonological recoding on analogical reasoning.

Moreover, the impact of language on analogy might also be located at the conceptual level of semantics rather than at the level of phonology: this would be consistent with data showing a positive influence of relational labeling on analogical reasoning (Christie \& Gentner, 2014; Gentner et al., 2009). In our study, we observed a positive association between vocabulary and morphosyntax in expression and analogical reasoning scores in typically developing children. This is in line with authors suggesting that analogical reasoning and language share a mutual influence, the former allowing for the development of the latter, which in turn favors the improvement of analogical reasoning ability (Gentner \& Christie, 2010). However, this relationship was not significant in children with DLD. It is, therefore, likely that the association between language and analogical reasoning is different in children with DLD than in their age-matched peers. It is also possible that correlations did not reach significance due to low power, and that such an influence takes place in children with DLD, with analogical reasoning participating to their language disorders, which in turn worsen their ability to perform analogies. Analogical scores are indeed associated with receptive vocabulary and expressive morphosyntax in these children, with correlations corresponding to 
medium effect sizes (although not significant due to a lack of power). Moreover, correlations indicated a medium association between response times in the analogical task and some language measures in both groups. Nevertheless, other studies are needed to confirm the nature of the relationship between language and analogical reasoning in children with DLD.

After having observed how performance is affected by external constraints, we were interested in testing how children react to different types of items in the task itself. Children are indeed impaired when they have to inhibit distractors sharing perceptual similarities with the target to focus on relational similarities. This has already been found in other studies. In a scene analogy task, Richland et al. (2006) found that children of 3 to 4 years old and of 6 to 7 years old tend to select the distractor that shares perceptual features with the target (see also Thibaut et al., 2010). Here, the impact of perceptual distraction was mainly observed for children with DLD. Other studies have shown that children with DLD heavily rely on perceptual cues and have difficulty in processing relational similarities alone. Leroy and colleagues found that children with DLD were more impaired by the decrease of relational similarity between the items of linguistic (Leroy, Maillart, \& Parisse, 2014) or non-linguistic (Leroy et al., 2012) analogies. Even in language development, these difficulties are visible: Leroy, Parisse, and Maillart (2014) used a priming task in which children have to produce a target sentence given a prime's structure, thus relying on structural alignment. These authors found that children with DLD had worse performance when the prime and the target shared no word (i.e., no perceptual similarity). These children are, therefore, particularly sensitive to perceptual features in analogical tasks. Our data confirm this dependence on perceptual features by showing that children with DLD are impaired when they have to resist perceptual distractors to reason about relational similarities. 
This difficulty in solving items in which perceptual distractors compete with the relational response can be related to inhibition. Inhibition reaction time was indeed found to influence performance in children with DLD. These findings are consistent with studies showing an influence of inhibition on analogical reasoning (Richland et al., 2006; Thibaut et al., 2010, 2011). Solving items with perceptual distractors seems, therefore, to be related to inhibition. However, the influence of inhibition was not detected for age-matched children. It is possible that, as these children were not as impacted by perceptual distraction as children with DLD, they did not need to rely on inhibition. However, it is also possible that our analysis failed to reach significance because of the low power and small sample size. Several studies have found an impairment of executive functions, including inhibition, in children with DLD (Cuperus et al., 2014; Im-Bolter et al., 2006; Kuusisto et al., 2017; Yang \& Gray, 2017). Given the influence of inhibition on analogical reasoning (Thibaut et al., 2011), this weakness in inhibition might be related to the analogical reasoning impairment observed in DLD, as well as to their difficulty in processing analogies when perceptual distractors are introduced. To our knowledge, our study is the first to report the influence of an inhibition measure on analogical reasoning in children with DLD. These findings, as well as the fact that children with DLD are more impacted by perceptual distraction than their peers, contribute to the idea that an inhibition deficit might explain the analogical reasoning impairment observed in DLD.

Analogical reasoning is related to nonverbal IQ and is influenced by inhibition in children with DLD, while it is linked to vocabulary and morphosyntax in typically-developing children (Table 4). Children with DLD might have insufficient conceptual skills to efficiently reason analogically (Table 1). These children are more reliant on perceptual features, which could explain the fact that inhibition is a significant predictor of analogical reasoning in these 
children. In contrast, typically-developing children seem to rely on conceptual skills to solve analogies; their conceptual skills might be sufficient to allow them to solve the task, and, also, to be less influenced by perceptual features. Consequently, they rely on less inhibition when reasoning analogically, or inhibition performance has less influence. It is also possible that these language variables support the development of nonverbal reasoning and that the language impairment observed in DLD leads to a decrease in nonverbal, and specifically analogical, reasoning (Botting, 2005). Interestingly, typically-developing children performed above average in receptive vocabulary (with a mean standardized score superior to 115). These children might have highly developed conceptual-semantic skills, probably due to high socioeconomic status (Maguire et al., 2018). This could have supported analogical reasoning and further contributed to the difference between the two groups. However, it is noteworthy that some of the correlations were of medium size, even if they did not reach statistical significance, most likely because of low power. It is, therefore, possible that other variables are associated with analogical reasoning in children with DLD, especially receptive vocabulary and expressive morphosyntax (Table 4). Conceptual skills might also be associated with analogical reasoning in these children, but additional studies with larger samples are needed to confirm these hypotheses.

This study has some limitations which need to be addressed. First, our sample size is relatively small, and the power of the analyses could have been enhanced with a larger number of participants. Some of the results are nonsignificant while corresponding to a moderate or high effect size. This is especially the case for the correlational analyses and for the influence of inhibition correct responses on analogical performance in children with DLD. It is likely that increasing the sample size, even by a few subjects, would have led to additional significant results. Moreover, our groups differed on socio-economic status. Even if 
this measure did not seem to influence analogical reasoning performance in our analyses, it is possible that this influence would have been significant with a higher statistical power. Socioeconomic status might, therefore, explain the deficit of children with DLD in analogical reasoning. Our design could also have been improved with an objective measure of the interference tasks: the tapping rate could have been measured with a keypad and a computer, thus providing information about the way participants were performing the interference task (Larsen \& Baddeley, 2003; Otsuka \& Osaka, 2015). It is possible that, in our study, the rate of the interference task was not held constant and that it did not cause as much interference as it should have. The use of articulatory suppression to examine the impact of language on analogical reasoning can also be questioned, especially given our unexpected findings on response times. More studies are required to fully understand the relationship between language and analogical reasoning, with designs that more precisely address the influence of phonological recoding and relational vocabulary on analogical reasoning in children with or without DLD. For example, a word describing the relation presented could be provided to analyze how this impacts performance in children with DLD, as it has already been shown to improve analogical reasoning in typically developing children (Christie \& Gentner, 2014; Gentner et al., 2009). Finally, our study highlighted a link between language, inhibition, and analogical reasoning; children with DLD are impaired when solving nonverbal analogies with perceptual distractors, which have to be inhibited. Even if the nature of this relationship needs to be clarified, our study is one of the only experiments to investigate the analogical reasoning impairment of children with DLD and to suggest possible explanations for this deficit. Our study also contributes new data about the relationship between language, executive functions, and analogical reasoning, which are all central functions of human cognition. 


\section{Conclusion}

To conclude, our study aimed at investigating the analogical reasoning impairment of children with DLD in relation to phonological recoding and inhibition. The analogical reasoning difficulties of children with DLD cannot be related to inefficient use of phonological recoding, as articulatory suppression does not impair the performance of either group. However, inhibition seems to play a role, as children, and especially children with DLD, are impaired when perceptual distractors are present among the solutions proposed. Inhibition also had a significant influence on performance for children with DLD. Even if our findings should be considered cautiously given the small number of participants, this study contributes some novel data about the links between language, analogical reasoning, and executive functioning. Given the influence that analogical reasoning has on language acquisition (Bybee, 2010; Gentner \& Christie, 2010), if we are to understand and improve language development in these children, the analogical reasoning impairment observed in DLD is of crucial interest.

\section{Acknowledgments}

We thank the children in the study, as well as the parents, teachers, and speech-language therapists. We are grateful to Milena Vezneva for the analogical task, and to Jade Philis and Mathilde Neefs for their help with data collection.

\section{Funding}

This work was supported by the FRESH fund of the Fonds National de la Recherche Scientifique. 


\section{Disclosure of interest}

The authors report no conflict of interest.

\section{References}

Baddeley, A. (1992). Working memory. Science, 255(5044), 556-559.

Baddeley, A. D., \& Larsen, J. D. (2007). The phonological loop unmasked? A comment on the evidence for a "perceptual-gestural" alternative. The Quarterly Journal of Experimental Psychology, 60(4), 497-504.

Baddeley, A. D., Thomson, N., \& Buchanan, M. (1975). Word length and the structure of short-term memory. Journal of Verbal Learning and Verbal Behavior, 14(6), 575-589.

Balthazar, C. H. (2003). The word length effect in children with language impairment. Journal of Communication Disorders, 36(6), 487-505.

Bishop, D. V., Snowling, M. J., Thompson, P. A., Greenhalgh, T., \& Catalise-2 Consortium. (2017). Phase 2 of CATALISE: A multinational and multidisciplinary Delphi consensus study of problems with language development: Terminology. Journal of Child Psychology and Psychiatry, 58(10), 1068-1080.

Botting, N. (2005). Non-verbal cognitive development and language impairment. Journal of Child Psychology and Psychiatry, 46(3), 317-326.

Bybee, J. (2010). Language, usage and cognition. Cambridge, England: Cambridge University Press.

Campoy, G., \& Baddeley, A. (2008). Phonological and semantic strategies in immediate serial recall. Memory, 16(4), 329-340.

Chevrie-Muller, C., Maillart, C., Simon, A.-M., \& Fournier, S. (2010). Batterie langage oral, langage écrit, mémoire, attention (2de éd.). Montreuil, France: ECPA.

Christie, S., \& Gentner, D. (2014). Language helps children succeed on a classic analogy task. Cognitive Science, 38(2), 383-397.

Coady, J. A., \& Evans, J. L. (2008). Uses and interpretations of non-word repetition tasks in children with and without specific language impairments (SLI). International Journal of Language \& Communication Disorders, 43(1), 1-40.

Cohen, J. (1988). Statistical power analysis for the behavioral sciences. Hillsdale, NJ: Lawrence Erlbaum Associates. 
Cuperus, J., Vugs, B., Scheper, A., \& Hendriks, M. (2014). Executive function behaviours in children with specific language impairment (SLI). International Journal of Developmental Disabilities, 60(3), 132-143.

Dunn, L. M., Theriault-Whalen, C. M., \& Dunn, L. M. (1993). Echelle de vocabulaire en images peabody. Toronto, Canada: Psychan.

Emerson, M. J., \& Miyake, A. (2003). The role of inner speech in task switching: A dual-task investigation. Journal of Memory and Language, 48(1), 148-168.

Fatzer, S. T., \& Roebers, C. M. (2012). Language and executive functions: The effect of articulatory suppression on executive functioning in children. Journal of Cognition and Development, 13(4), 454-472.

Gentner, D., \& Christie, S. (2010). Mutual bootstrapping between language and analogical processing. Language and Cognition, 2(2), 261-283.

Gentner, D., Simms, N., \& Flusberg, S. (2009). Relational language helps children reason analogically. In Proceedings of the 31st Annual Conference of the Cognitive Science Society (pp. 1054-1059). Cognitive Science Society.

Gentner, D., \& Smith, L. (2012). Analogical reasoning. In V. S. Ramachandran (Ed.), Encyclopedia of Human Behavior (2nd Ed.) (pp. 130-136). Oxford, UK: Elsevier.

Geurten, M., Catale, C., \& Meulemans, T. (2016). Involvement of executive functions in children's metamemory. Applied Cognitive Psychology, 30(1), 70-80.

Hill, E. L. (2001). Non-specific nature of specific language impairment: A review of the literature with regard to concomitant motor impairments. International Journal of Language \& Communication Disorders, 36(2), 149-171.

Hofstadter, D., \& Sander, E. (2013). L'Analogie: Cour de la pensée. Paris, France: Odile Jacob Sciences.

Im-Bolter, N., Johnson, J., \& Pascual-Leone, J. (2006). Processing limitations in children with specific language impairment: The role of executive function. Child Development, 77(6), 1822-1841.

Kemény, F., \& Lukács, Á. (2010). Impaired procedural learning in language impairment: Results from probabilistic categorization. Journal of Clinical and Experimental Neuropsychology, 32(3), 249-258.

Khomsi, A. (2001). ELO: Evaluation du langage oral. Paris, France: ECPS.

Kotovsky, L., \& Gentner, D. (1996). Comparison and categorization in the development of relational similarity. Child Development, 67(6), 2797-2822. 
Krzemien, M., Jemel, B., \& Maillart, C. (2017). Analogical reasoning in children with specific language impairment: Evidence from a scene analogy task. Clinical Linguistics \& Phonetics, 31, 573-588.

Kuusisto, M. A., Nieminen, P. E., Helminen, M. T., \& Kleemola, L. (2017). Executive and intellectual functioning in school-aged children with specific language impairment. International Journal of Language \& Communication Disorders, 52(2), 127-136.

Larsen, J. D., \& Baddeley, A. (2003). Disruption of verbal STM by irrelevant speech, articulatory suppression, and manual tapping: Do they have a common source? The Quarterly Journal of Experimental Psychology Section A, 56(8), 1249-1268.

Lecocq, P. (1996). L'ECOSSE: Une épreuve de compréhension syntaxico-sémantique. Villeneuve-d'Ascq, France : Presses Universitaires du Septentrion.

Leonard, L. B. (2014). Children with specific language impairment. Cambridge, MA: The MIT Press.

Leroy, S., Maillart, C., \& Parisse, C. (2014). Analogical mapping across modalities in children with specific language impairment (SLI). Research in Developmental Disabilities, 35(9), 2158-2171.

Leroy, S., Parisse, C., \& Maillart, C. (2012). Analogical reasoning in children with specific language impairment. Clinical Linguistics \& Phonetics, 26(4), 380-395.

Leroy, S., Parisse, C., \& Maillart, C. (2014). Le manque de généralisation chez les enfants dysphasiques: Une étude longitudinale. ANAE, 131, 1-9.

Lidstone, J. S., Meins, E., \& Fernyhough, C. (2010). The roles of private speech and inner speech in planning during middle childhood: Evidence from a dual task paradigm. Journal of Experimental Child Psychology, 107(4), 438-451.

Lidstone, J. S., Meins, E., \& Fernyhough, C. (2012). Verbal mediation of cognition in children with specific language impairment. Development and Psychopathology, 24(2), 651-660.

Logie, R. H., Della Sala, S., Wynn, V., \& Baddeley, A. D. (2000). Visual similarity effects in immediate verbal serial recall. The Quarterly Journal of Experimental Psychology Section A, 53(3), 626-646.

Lum, J. A., \& Conti-Ramsden, G. (2013). Long-term memory: A review and meta-analysis of studies of declarative and procedural memory in specific language impairment. Topics in Language Disorders, 33(4), 282-297. 
Maguire, M. J., Schneider, J. M., Middleton, A. E., Ralph, Y., Lopez, M., Ackerman, R. A., \& Abel, A. D. (2018). Vocabulary knowledge mediates the link between socioeconomic status and word learning in grade school. Journal of Experimental Child Psychology, $166,679-695$.

Masterson, J. J., Evans, L. H., \& Aloia, M. (1993). Verbal analogical reasoning in children with language-learning disabilities. Journal of Speech and Hearing Research, 36, 7682.

Mussweiler, T., \& Epstude, K. (2009). Relatively fast! Efficiency advantages of comparative thinking. Journal of Experimental Psychology: General, 138(1), 1-21.

Niemi, J., Gundersen, H., Leppäsaari, T., \& Hugdahl, K. (2003). Speech lateralization and attention/executive functions in a Finnish family with specific language impairment (SLI). Journal of Clinical and Experimental Neuropsychology, 25(4), 457-464.

Nippold, M. A., Erskine, B. J., \& Freed, D. B. (1988). Proportional and functional analogical reasoning in normal and language-impaired children. Journal of Speech \& Hearing Disorders, 53, 440-448.

Norris, D., Butterfield, S., Hall, J., \& Page, M. P. (2018). Phonological recoding under articulatory suppression. Memory \& Cognition, 46(2), 173-180.

Otsuka, Y., \& Osaka, N. (2015). High-performers use the phonological loop less to process mental arithmetic during working memory tasks. The Quarterly Journal of Experimental Psychology, 68(5), 878-886.

Poloczek, S., Henry, L. A., Messer, D. J., \& Büttner, G. (2019). Do children use different forms of verbal rehearsal in serial picture recall tasks? A multi-method study. Memory.

Psychology Software Tools, Inc. (2012). E-Prime 2.0. Retrieved from http://www.pstnet.com

R Development Core Team. (2016). R: A language and environment for statistical computing. Vienna, Austria: R Foundation for Statistical Computing.

Ramus, F., Marshall, C. R., Rosen, S., \& van der Lely, H. K. (2013). Phonological deficits in specific language impairment and developmental dyslexia: Towards a multidimensional model. Brain, 136(2), 630-645.

Rao, K. V., \& Baddeley, A. (2013). Raven's matrices and working memory: A dual-task approach. The Quarterly Journal of Experimental Psychology, 66(10), 1881-1887. 
Richland, L. E., Morrison, R. G., \& Holyoak, K. J. (2006). Children's development of analogical reasoning: Insights from scene analogy problems. Journal of Experimental Child Psychology, 94(3), 249-273.

Stigler, J. W., Lee, S. Y., \& Stevenson, H. W. (1986). Digit memory in Chinese and English: Evidence for a temporally limited store. Cognition, 23(1), 1-20.

Sullivan, J., \& Barner, D. (2013). How are number words mapped to approximate magnitudes? The Quarterly Journal of Experimental Psychology, 66(2), 389-402.

Thibaut, J.-P., French, R., \& Vezneva, M. (2010). The development of analogy making in children: Cognitive load and executive functions. Journal of Experimental Child Psychology, 106(1), 1-19.

Thibaut, J. P., French, R. M., Vezneva, M., Gérard, Y., \& Glady, Y. (2011). Semantic analogies by young children: Testing the role of inhibition. In B. Kokinov, A. Karmiloff-Smith, \& N. J. Nersessian (Eds.), European Perspectives on Cognitive Science (pp. 136-140). Sofia, Bulgaria: New Bulgarian University Press.

Tomblin, J. B., Records, N. L., Buckwalter, P., Xuyang, Z., Smith, E., \& O'Brien, M. (1997). Prevalence of specific language impairment in kindergarten children. Journal of Speech, Language, and Hearing Research, 40, 1245-1260.

Vezneva, M. (2011). Développement du raisonnement analogique : rôle de la composante exécutive d'inhibition (Unpublished doctoral dissertation). Université de Bourgogne, Dijon, France.

Vugs, B., Hendriks, M. P. H., Cuperus, J., \& Knoors, H. (2017). Developmental associations between working memory and language in children with specific language impairment: A longitudinal study. Journal of Speech, Language, and Hearing Research, 60(11), 2384-2394.

Waltz, J. A., Lau, A., Grewal, S. K., \& Holyoak, K. J. (2000). The role of working memory in analogical mapping. Memory \& Cognition, 28(7), 1205-1212.

Wechsler, D., \& Naglieri, J. (2009). Echelle non verbale d'intelligence de Wechsler. Montreuil, France: ECPA.

Yang, H.-C., \& Gray, S. (2017). Executive function in preschoolers with primary language impairment. Journal of Speech, Language, and Hearing Research, 60, 379-392.

Zelaznik, H. N., \& Goffman, L. (2010). Generalized motor abilities and timing behavior in children with specific language impairment. Journal of Speech, Language, and Hearing Research, 53(2), 383-393. 